\title{
Micro/Nanoscale Manufacture of Advanced Materials and an Exploration of Their Properties
}

\author{
Kausala Mylvaganam $\mathbb{D}^{1},{ }^{1}$ Haihui Ruan, ${ }^{2}$ Weidong Liu, ${ }^{3}$ and Chengyuan Wang ${ }^{4}$ \\ ${ }^{1}$ University of Sydney, Camperdown, NSW 2006, Australia \\ ${ }^{2}$ Department of Mechanical Engineering, The Hong Kong Polytechnic University, Hung Hom, Kowloon, Hong Kong \\ ${ }^{3}$ University of New South Wales, Sydney, Australia \\ ${ }^{4}$ Swansea University, Swansea, UK
}

Correspondence should be addressed to Kausala Mylvaganam; kausala.mylvaganam@gmail.com

Received 13 June 2018; Accepted 14 June 2018; Published 30 July 2018

Copyright ( $) 2018$ Kausala Mylvaganam et al. This is an open access article distributed under the Creative Commons Attribution License, which permits unrestricted use, distribution, and reproduction in any medium, provided the original work is properly cited.

This special issue is focused on the manufacturing of advanced materials at the microscale and nanoscale and an exploration of their properties. Manufacturing at the micro/ nanoscale is already an extensive field, leading to the production of improved materials and new products. A significant number of papers were received, and after going through a comprehensive peer review process, seven papers were selected to be included in this special issue. Each paper analyses different properties of nanomaterials, most of which are carbon-based. The papers published in this special issue highlight the original work related to advanced materials and will give the readers a new insight.

In the paper by C.-M. Cheng et al., carbon-based nanofluids are synthesized by a vortex trap method (VTM), with an oxyacetylene flame using different flow rate ratios of oxygen and acetylene, as the carbon source. By investigating the morphology, structure, particle size, stability, and basic physicochemical characteristics of the obtained carbon-based nanomaterials (CBNMs), the authors have identified the flow rate that gives the highest stability CBNMs.

In the paper by X. Wei et al., an infrared-laser-irradiated field-emission electronic pulse system based on carbon nanotube cathodes is demonstrated. Comparing to the present field-emission system based on metallic tips, nanotubes have much lower manufacturing cost yet render much larger tip aspect ratio and stronger field enhancement. Their results extended conventional electron sources and provided a possibility to realize ultrashort electronic pulses as a highfrequency electron source.

In the paper by R. L. Garnica-Gutiérrez et al., acidfunctionalized and poly-citric acid- (PCA-) polymerized carbon nanotubes (CNTs) were placed in contact with the extracellular membrane (ECM) of mesenchymal stem cells (MSC). The authors then examined their effects on the MSC in various biological processes, showing that PCApolymerized CNTs are less cytotoxic and lead to less apoptotic cell death. The effects of nitrogen-doped CNTs (CNxs) are also reported. Undoped CNTs tend to stimulate cell proliferation, whereas CNxs promote the apoptotic behavior. This pioneering work provides useful guidance for further study of the role of the CNT-ECM interaction on the CNT-MSC structures which are crucial for tissue grafting.

In the paper by $\mathrm{Z}$. Wu et al., vibrations are studied for double-layer graphene sheets (DLGSs) in thermal environments. The vibration equation is derived by incorporating the nonlocal elastic theory into classical plate theory (CLPT). The element-free method is then employed to implement the vibration analyses. The technique used is validated in comparison with existing models in benchmark cases. Subsequently, the authors have conducted a comprehensive study of DLGS vibration and quantified the effects of boundary conditions, nonlocal parameter, elastic foundation parameter, and temperature.

In the paper by $\mathrm{B}$. Chaitongrat and $\mathrm{S}$. Chaisitsak, a room temperature LPG sensor is achieved based on $\alpha-\mathrm{Fe}_{2} \mathrm{O}_{3} / \mathrm{CNT}$ 
(carbon nanotube) nanocomposite films fabricated via the $\mathrm{Fe}$ catalyst particles on CNTs, which were synthesized by chemical vapor deposition (CVD) and then annealed to create $\alpha-\mathrm{Fe}_{2} \mathrm{O}_{3}$. These simple and cost-efficient methods are suitable for large-scale manufacturing. The structures, surface morphologies, and LPG response are then studied for the films via Raman spectroscopy, XPS analysis, and FE-SEM or AFM. It is shown that the LPG sensors are characterized with high sensitivity and quick response.

In the paper by $\mathrm{X}$. Dong et al., submicron grooves are machined on the surface of crystalline nickel phosphide-a commonly used coating material for precision glass moulding. These subwavelength-scale features could later be replicated onto the surface of optical elements for modulating light path. To achieve the extreme of quality of machining, the nanoscale manufacturing defects such as blurs and pits are studied in detail.

In the paper by $S$. Kasmi et al., self-organized silica nanosphere (NS) layers are fabricated by a facile spin coating process. The authors further explore the role of the NS diameter, the spin coating acceleration, and the volume fraction in the formation and organization of NS during the coating process. The competition between the sedimentation and viscosity of the solution is recognized as the key factors that control the layer thickness. By selecting proper parameters, one can thus choose to form either a single- or double-layer of closely packed NS.

\section{Acknowledgments}

We owe the authors of this special issue, our appreciation for having contributed their research papers for publication. We also thank our expert reviewers for their expeditive and constructive comments and criticisms during their review processes.

Kausala Mylvaganam Haihui Ruan Weidong Liu Chengyuan Wang 


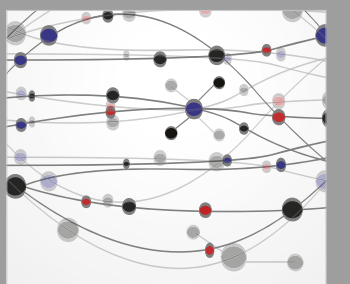

The Scientific World Journal
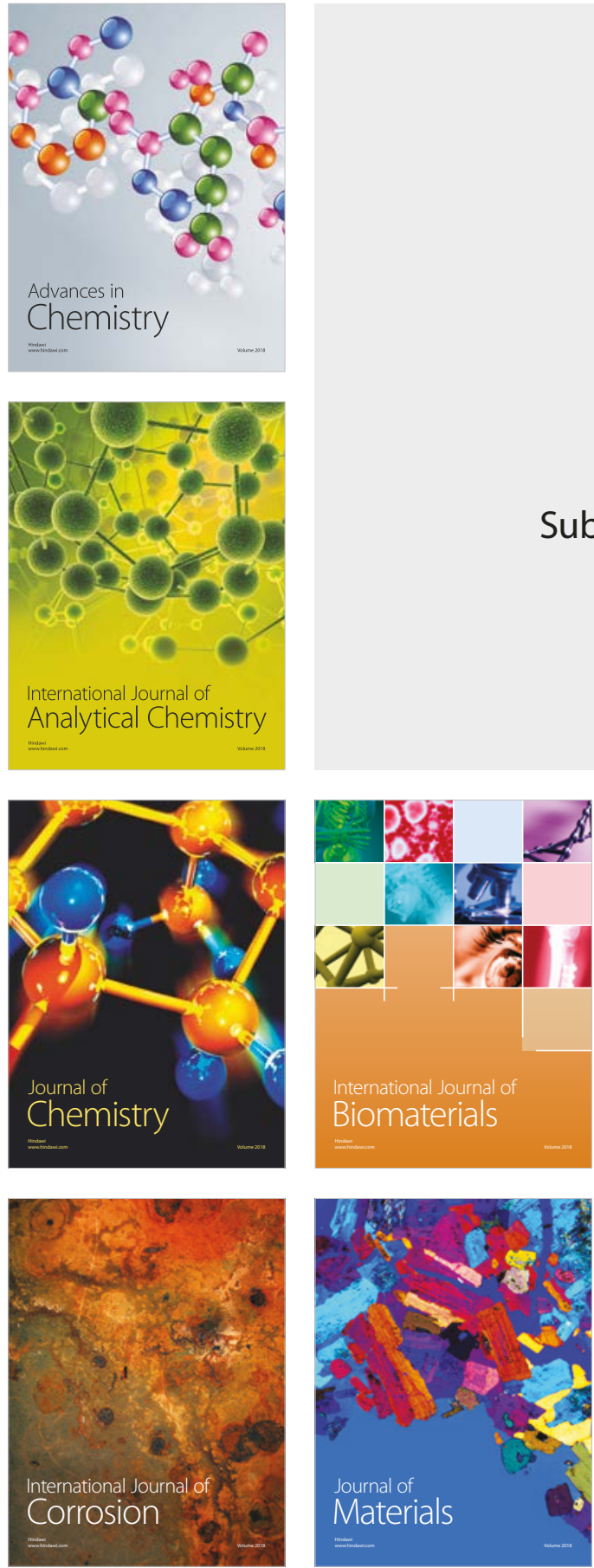

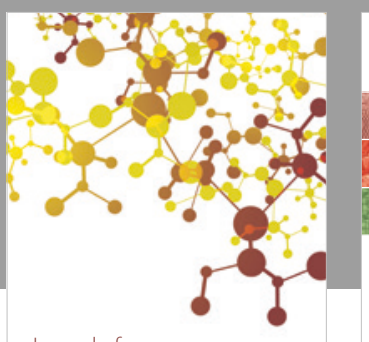

Journal of

Applied Chemistry
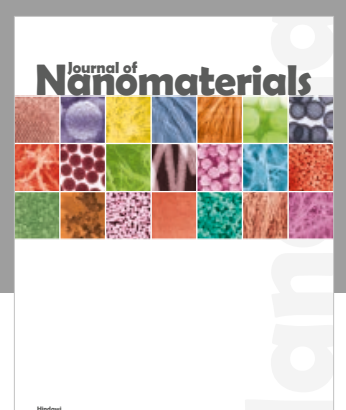

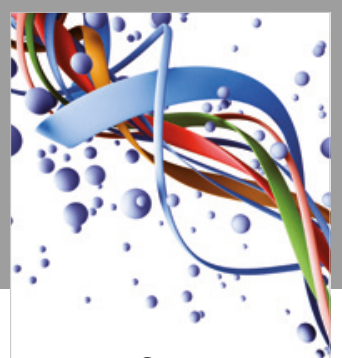

Scientifica

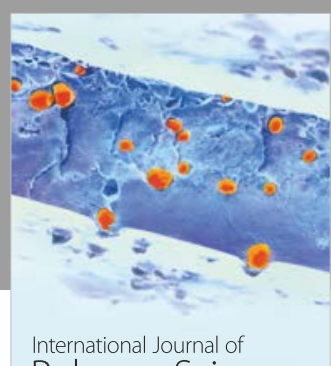

Polymer Science

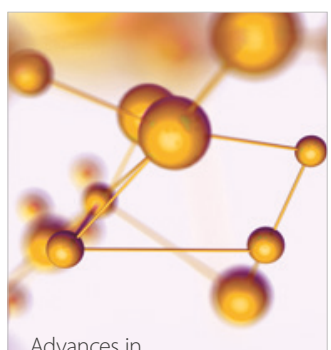

Physical Chemistry
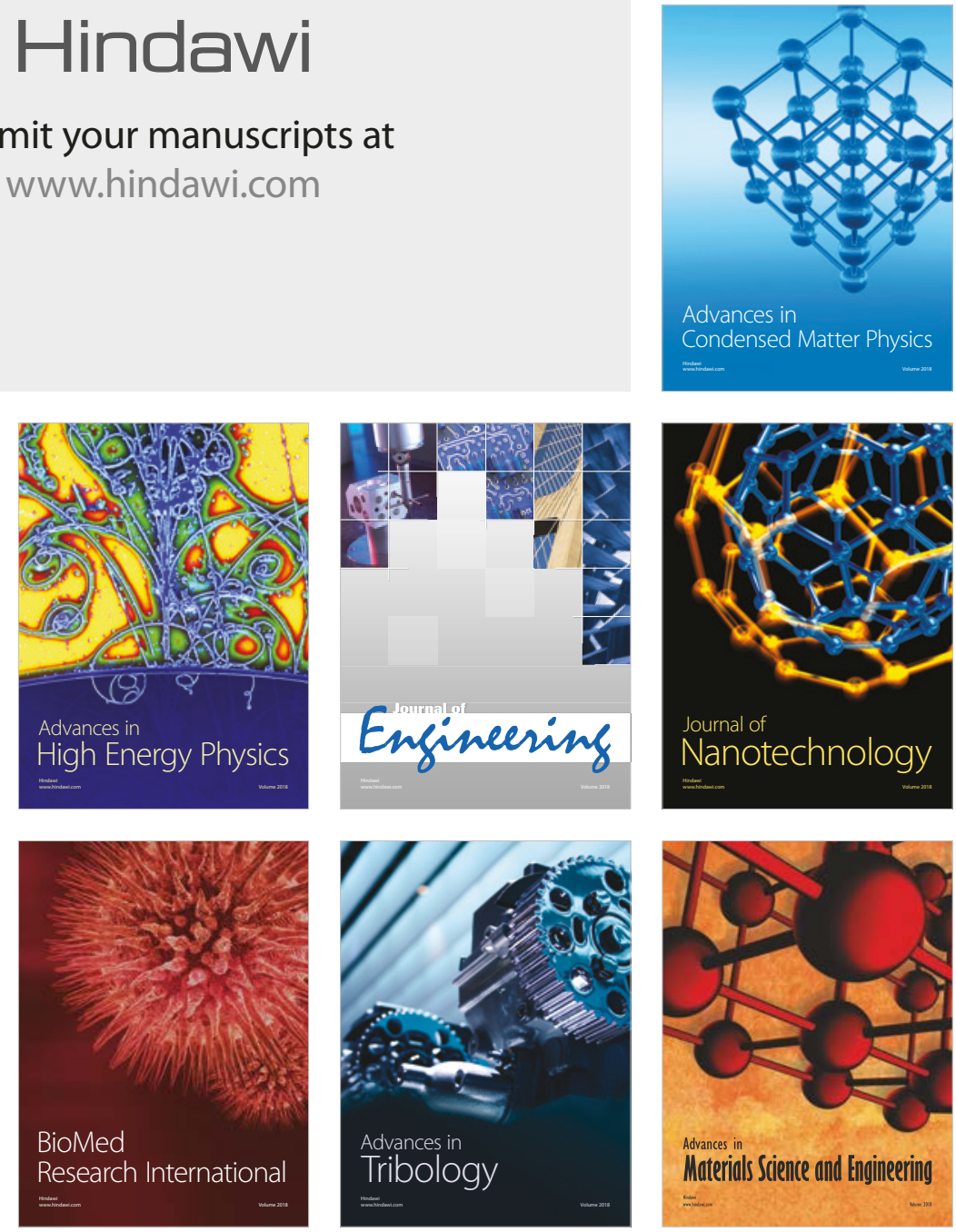